\title{
A Multicenter, Randomized, Open-Label Trial Comparing the Efficacy and Safety of Monoclonal Anti-Rh (D) Immunoglobulin with Polyclonal Anti-Rh (D) Immunoglobulin for the Prevention of Maternal Rh-Isoimmunization
}

\author{
Anahita R. Chauhan ${ }^{1} \cdot$ Yogeshwar S. Nandanwar $^{2} \cdot$ Aruna Ramaiah $^{3} \cdot$ Kanan A. Yelikar $^{4} \cdot$ M. D. Rashmi ${ }^{5}$. \\ Rekha Sachan $^{6} \cdot$ Rahul V. Mayekar $^{2} \cdot$ Yamini N. Trivedi $^{7} \cdot$ Gopalkrishna V. Paradkar $^{8} \cdot$ Kiran P. Patole $^{9}$
}

Received: 12 December 2018 / Accepted: 29 April 2019 / Published online: 3 June 2019

(c) Federation of Obstetric \& Gynecological Societies of India 2019

\begin{abstract}
Objectives To compare the efficacy and safety of monoclonal anti-Rhesus (anti-D) immunoglobulin (IgG) with polyclonal anti-D IgG in the prevention of maternal Rh-isoimmunization.

Methods This was a randomized, multicenter, open-label, comparative clinical trial conducted in the obstetric in-patient departments of nine tertiary care hospitals in India. 206 Rhesus (D)-negative women, not sensitized to Rh antigen, and delivering Rh positive babies, received postpartum intramuscular administration of monoclonal or polyclonal anti-D IgG. The main outcome measures were the proportion of subjects protected from $\mathrm{Rh}$-isoimmunization, identified by a negative indirect Coombs test (ICT) result, at day 180 after anti-D IgG administration, and incidence of adverse events.

Results 105 subjects were randomized to the monoclonal group and 101 to the polyclonal group. 94 from the monoclonal group had a negative ICT result and none had a positive ICT result at day 180, whereas 87 from the polyclonal group had a negative ICT result and one had a positive ICT result; the rest (11 and 13 subjects respectively) were lost to follow-up. A total of 5 adverse events were reported ( 3 in the monoclonal group and 2 in the polyclonal group); only one of these was serious. All the adverse events were judged to be unrelated to the interventional drug. None of the subjects in the monoclonal group developed immunogenic reaction to the monoclonal anti-D.

Conclusion The efficacy and safety of the monoclonal preparation of anti-D was comparable to the polyclonal preparation of anti-D when used in the prevention of maternal Rh-isoimmunization.
\end{abstract}

Trial registration Clinical Trial Registration Number: CTRI/2015/09/006172.

Keywords Rhesus $\cdot$ Immunoglobulin $\cdot$ Monoclonal $\cdot$ Isoimmunization

Anahita R. Chauhan is a Professor and Unit Head, Department of Obstetrics and Gynaecology. Yogeshwar S. Nandanwar is a Professor and Head, Department of Obstetrics and Gynaecology. Aruna Ramaiah is a Professor and Superintendent. Kanan A. Yelikar is a Professor, Department of Obstetrics and Gynaecology. M. D. Rashmi is a Senior Consultant. Department of Obstetrics and Gynaecology. Rekha Sachan is a Professor, Department of Obstetrics and Gynaecology. Rahul V. Mayekar is a Associate Professor, Department of Obstetrics and Gynaecology. Yamini N. Trivedi is a Professor and Head, Department of Obstetrics and Gynaecology. Gopalkrishna V. Paradkar is a Professor and Head, Department of Obstetrics and Gynaecology. Kiran P. Patole is a Professor and Head, Department of Obstetrics and Gynaecology.

Anahita R. Chauhan

anahitachauhan@gmail.com

Extended author information available on the last page of the article

\section{Introduction}

The clinical practice of passive immunization of Rhesus $(\mathrm{Rh})$ negative pregnant women with anti-Rh immunoglobulin $(\mathrm{IgG})$ for the prevention of sensitisation to the Rh (D) antigen began in the 1960s, when multiple studies reported about its effectiveness and possible mechanisms of action [1-3]. The most important sensitizing event for Rh-negative women occurs at the end of the pregnancy, with detachment of the placenta during delivery. Prophylaxis with anti-D $\mathrm{IgG}$ induces a strong immunosuppressive effect, although its exact mechanism is not fully understood $[3,4]$. The proposed mechanisms include accelerated clearance of $\mathrm{Rh}$ positive cells, epitope masking, inhibition due to antibodies 
against Fc $\gamma$ RIIB or anti-idiotype, inhibition of immature dendritic cells, and inhibition of B-cell clones specific for the Rh antigen. Accelerated destruction of Rh-positive red blood cells is the most widely accepted main mechanism of action $[3,4]$. The incidence of postpartum anti-D sensitization has reduced from $13-19$ to $0.9-1.8 \%$ with postpartum immunoprophylaxis and further to $0.1-0.3 \%$ with addition of antenatal immunoprophylaxis [5-7]. With these high success rates, effectiveness over a period of 12 weeks [4] and safe profile, anti-D IgG exemplifies a remarkable success story in the field of obstetrics and preventive care. Owing to these dramatic results with anti-D, systematic anti-D prophylaxis in all Rh-negative pregnant women was proposed in the 1970s and has become part of standard guidelines across the world $[1,8,9]$.

The conventional polyclonal anti-D IgG is produced by fractionation of $\mathrm{IgG}$ from pooled plasma of donors who are mostly men, deliberately immunized with Rh-positive red cells [1]. The manufacture of polyclonal anti-D IgG is thus limited by the availability of suitable human plasma donors. Monoclonal anti-D IgG produced using hybridoma and recombinant DNA technologies overcome this limitation and provide a virtually unlimited supply of the antibody. The available monoclonal anti-D has been demonstrated to have similar physicochemical and biological properties to conventional polyclonal anti-D.

This study aimed to compare the efficacy and safety of this monoclonal anti-D preparation with that of conventional polyclonal anti-D when used for postpartum immunoprophylaxis.

\section{Materials and Methods}

\section{Study Design}

This was a randomized, controlled, open, multicenter trial comparing monoclonal anti-D preparation with conventional polyclonal anti-D preparation. The overall study was designed as per the European Medicines Agency "Guideline on the clinical investigation of human anti-D immunoglobulin for intravenous and/or intramuscular use - CPMP/ BPWG/575/99 Rev. 1" [10]. The trial was conducted at obstetric in-patient departments of 9 tertiary care hospitals in India. The trial protocol (code: BSV/Rh-ANTI D/13) was approved by the Indian drug regulatory authority and the institutional ethics committees of all the participating centres. The trial was performed in accordance with the principles of the Declaration of Helsinki, the International Conference on Harmonization Guidelines for Good Clinical Practice, and local regulatory requirements. All participants were explained about the study and the need for follow up, and provided voluntary, written, informed consent.

\section{Study Participants}

Rh-negative pregnant women delivering a Rh-positive baby, with a negative ICT test result were eligible for the study. The main exclusion criteria were positive ICT test result at baseline, Rh-negative blood group of husband/partner, history of incompatible blood transfusion, history of allergic reaction to IgGs, anticipated requirement of blood transfusion after delivery and diagnosis of abruptio placentae, placenta previa or intrauterine death. The present study was envisaged as a study with two groups in 1:1 ratio with a sample size of 100 subjects per group. Up to $1.8 \%$ of women develop anti-D antibodies despite post-natal anti-D administration [5]. Therefore, if there is truly no difference between the standard and experimental treatment, then 176 subjects were required to be $80 \%$ sure that the upper limit of one-sided $95 \%$ confidence interval will exclude a difference of more than $5 \%$.

\section{Study Randomization}

Women were randomly assigned in a 1:1 ratio, to either the monoclonal or polyclonal anti-D groups, using a computer-generated randomization code. The codes were provided to the sites in sealed envelopes.

\section{Intervention}

The subjects received either monoclonal anti-D (Rhoclone ${ }^{\circledR}$, Bharat Serums and Vaccines Ltd.) or polyclonal anti-D (RhoGAM ${ }^{\circledR}$, Johnson \& Johnson) at a dose of $300 \mathrm{mcg}$ (1500 IU) within $72 \mathrm{~h}$ of delivery. The dose was fixed at $300 \mathrm{mcg}$, since a dose of $300 \mathrm{mcg}$ (1500 IU) of anti-D IgG protects against $30 \mathrm{~mL}$ of whole blood [9] and literature data report that the feto-maternal hemorrhage (FMH) exceeding $30 \mathrm{~mL}$ is exceedingly rare $(0.2-0.3 \%$ pregnancies) $[4,11]$. Cases where investigators suspected FMH $>30 \mathrm{ml}$ were not considered for the study.

\section{Study Procedure}

Each eligible subject received a single intramuscular injection of anti-D IgG within $72 \mathrm{~h}$ of delivery. Blood samples were collected before study drug administration (baseline) and at 72 h, 90 days and 180 days from the anti-D administration, as recommended by the European Medicines Agency guidelines. ICT was performed on all samples, while testing for anti-drug antibodies was performed on baseline, day 90 and day 180 samples. Adverse events were recorded throughout the study. 


\section{Study Outcomes}

The primary efficacy variable was the proportion of subjects with a negative ICT result on day 180 following administration of anti-D. ICT is used to detect circulating antibodies to red cell antigens. A positive ICT result in a subject at day 180 , who had negative ICT result before administration of anti-D would indicate that the subject got immunized to Rh antigen. ICT results at $72 \mathrm{~h}$ and Day 90 were also assessed, however since anti-D IgG from administered anti-D injection is present in detectable quantities up to 12 weeks [5] and since it is not possible to distinguish between administered and immune anti-D IgG, these results were considered as supportive evidence and were not carried forward for Day 180 . Only serial rise in titers was considered as positive result.

The safety variables were incidence of adverse events, including the injection site reactions in both groups, and incidence of immunogenicity (development of anti-drug antibodies) in the monoclonal group.

\section{Statistical Analysis}

The results were analyzed to explore the non-inferiority of monoclonal anti-D with polyclonal anti-D for the primary efficacy variable. The results were analyzed by using the Pearson's Chi square test.

\section{Results}

\section{Baseline Characteristics}

The trial was conducted between October 12, 2015 and December 21, 2016. A total of 229 women were screened across the 9 study centres, of whom 206 were randomized and received one of the study drugs: 105 received monoclonal anti-D and 101 received polyclonal anti-D. The trial and participant flow is shown in Fig. 1.

Demographics and baseline characteristics were comparable between the two treatment groups (Table 1). The ITT/ safety population included all 206 subjects who received either of the study drugs and the per protocol (PP) population included 204 (99\%) subjects. Two subjects enrolled into the study had a protocol deviation and hence were excluded from the PP population. However, since the difference between ITT and PP populations was minor, only ITT analysis was performed. Out of 206 randomized subjects, $194(94.1 \%)$ subjects completed day 90 visit whereas 182

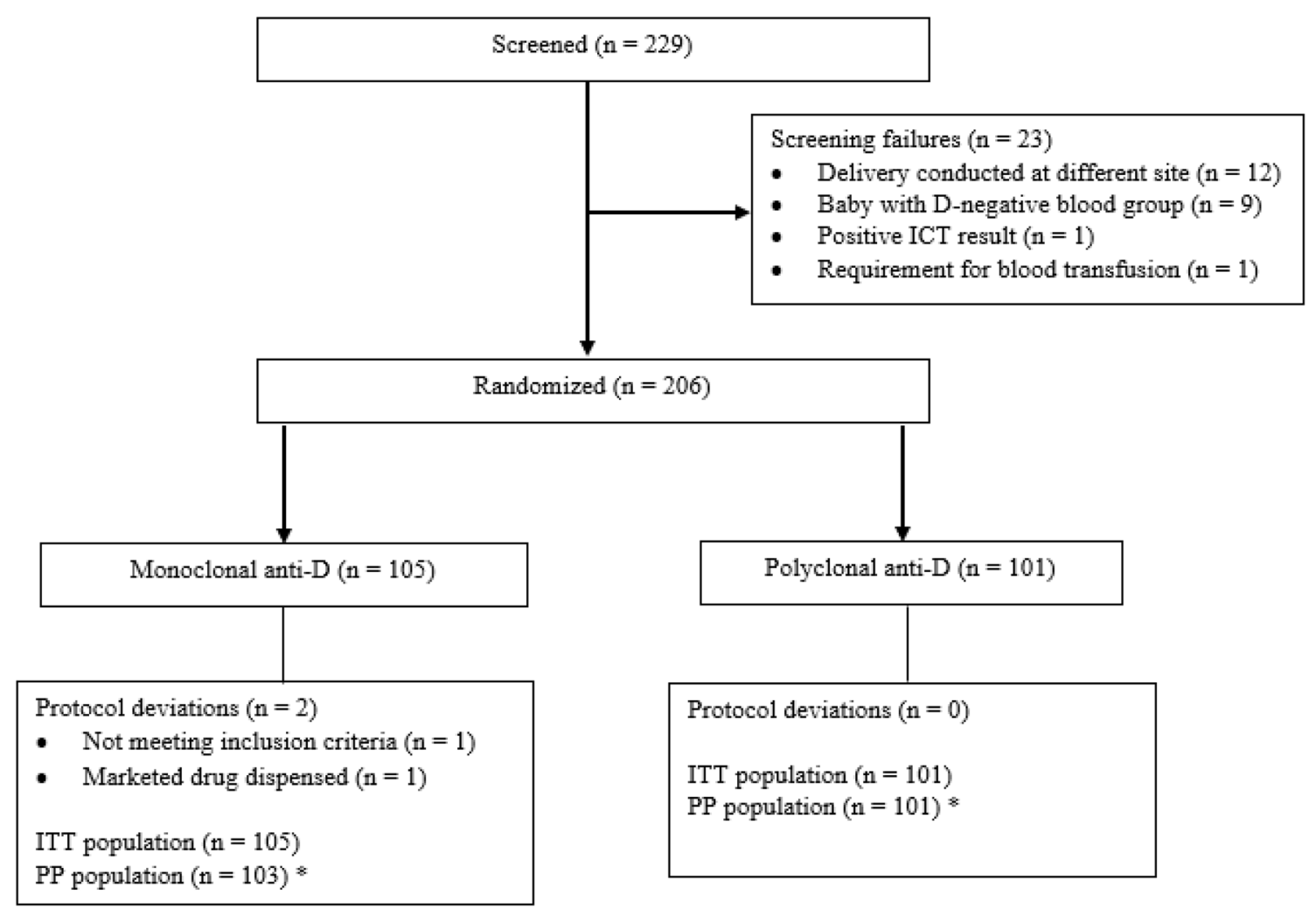

* Since the difference between ITT and PP population was minor, only ITT analysis was performed

Fig. 1 Trial and participant flow 
Table 1 Demographics and baseline characteristics

\begin{tabular}{lll}
\hline & Monoclonal anti-D & Polyclonal anti-D \\
\hline Subjects randomized $(n)$ & 105 & 101 \\
Age (mean \pm SD) & $24.77 \pm 4.01$ & $24.75 \pm 3.88$ \\
Type of delivery $(n)$ & & \\
Vaginal & 74 & 62 \\
LSCS & 28 & 37 \\
Forceps & 3 & 2 \\
Subjects who completed day 90 visit $[n(\%)]$ & $101(96.2)$ & $93(92.1)$ \\
Subjects who completed day 180 visit $[n(\%)]$ & $94(89.5)$ & $88(87.1)$ \\
\hline
\end{tabular}

$S D$ standard deviation, $L S C S$ lower segment caesarean section
(88.3\%) subjects completed day 180 visit. One subject died during the study due to an event unrelated to the study. No subject was discontinued due to safety reasons. Among the randomized subjects, $10.5 \%$ subjects from the monoclonal group and $12.9 \%$ subjects from the polyclonal group were lost to follow up before study completion.

\section{Efficacy Endpoints}

At Day 90, 2 subjects (2\%) from the polyclonal group and none from the monoclonal group had a positive ICT result. At Day 180, 1 subject from the polyclonal group and none from the monoclonal group had a positive ICT result. At Day 90, a negative ICT, indicating absence of $\mathrm{Rh}$ immunization was seen in $96.2 \%$ subjects in monoclonal group and $90.1 \%$ subjects in the polyclonal group.

In the ITT population, the primary efficacy parameter (negative ICT result) at day 180 after administration of antiD was reported in $89.5 \%$ in the monoclonal group and $86.1 \%$ in the polyclonal group.

The $p$ values for ICT results using Chi square test for day $90(p=0.13)$ and day $180(p=0.30)$ were not statistically significant and, thus, demonstrated similar efficacy between the monoclonal and polyclonal anti-D groups (Table 2).

\section{Safety Outcomes}

Five AEs were reported by 5 subjects; 3 subjects from the monoclonal anti-D group and 2 subjects from the polyclonal anti-D group (Table 3). Out of the 3 AEs reported in monoclonal group, 2 were mild and 1 was severe and both AEs reported in polyclonal group were mild. The severe AE reported in monoclonal group pertained to a 22-year old primigravida who was being medically managed for severe pre-eclampsia and severe oligohydramnios with intrauterine growth retardation. She subsequently underwent emergency LSCS for fetal distress following which she received monoclonal anti-D IgG. However, two days later, she developed
Table 2 Efficacy data-indirect Coombs test results

\begin{tabular}{lccl}
\hline Time-point and result & $\begin{array}{l}\text { Monoclo- } \\
\text { nal anti-D } \\
(n=105)\end{array}$ & $\begin{array}{l}\text { Polyclonal } \\
\text { anti-D } \\
(n=101)\end{array}$ & $\begin{array}{l}P \text { value }^{\mathrm{a}} \\
\text { (Chi square }_{\text {test })}\end{array}$ \\
\hline Day 90 & & & \\
Positive & 0 & 2 & 0.08 (NS) \\
Negative & 101 & 91 & \\
LTF & 4 & 8 & 0.46 (NS) \\
Day 180 & & & \\
Positive & 0 & 1 & \\
Negative & 94 & 87 & \\
LTF & 11 & 13 & \\
\hline
\end{tabular}

${ }^{\mathrm{a}}$ Calculated for ITT population with lost to follow-up patients considered as failure of therapy; LTF lost to follow-up, NS not statistically significant

Table 3 Safety data-adverse events

\begin{tabular}{lll}
\hline Adverse event & $\begin{array}{l}\text { Monoclonal anti-D } \\
\text { group }\end{array}$ & $\begin{array}{l}\text { Polyclonal } \\
\text { anti-D group }\end{array}$ \\
\hline Anemia & 0 & 2 \\
Body ache & 1 & 0 \\
Itching & 1 & 0 \\
Cerebral infarct $^{\mathrm{a}}$ & 1 & 0
\end{tabular}

${ }^{a}$ Outcome was death; unrelated to the study. All adverse events were judged to be unrelated to study treatment

cerebral infarction and 8 days later succumbed, despite intensive care management. The event was considered unrelated to the study drug, but due to the concurrent illness. All other AEs resolved without any sequelae/complications. All the AEs were judged to be unrelated to the study drugs.

\section{Immunogenicity}

The immunogenicity testing (test to demonstrate generation of antibodies) in the monoclonal anti $\mathrm{D}$ group revealed that none of the subjects developed antibodies against monoclonal anti-D. 


\section{Discussion}

The estimated worldwide prevalence of Rh disease is 276 per 100,000 live births [9]. The conventional polyclonal preparation of anti-D IgG, with its high success rate and safety profile has been the mainstay in the prevention of Rh disease for over 5 decades now. In recent times, however, the commercial availability of this IgG preparation has been affected, primarily due to limited availability of this hyperimmune plasma. There now exists a world-wide shortage of polyclonal anti-D Ig, leading to an increased cost over the monoclonal anti-D, a situation not anticipated to improve [4, 11]. Additionally, there is increasing concern about the risks of transmission and newly emerging viruses, especially since in most countries all Rh-negative pregnant women are offered anti-D antenatally, irrespective of the Rh status of the foetus [1].

Manufacturing monoclonal and recombinant anti-D antibodies using modern biotechnology methods started over three decades ago. These specific anti-D antibodies originate from human B cells and should be able to overcome the concerns with respect to safety and supply of anti-D $[4,11]$. The technology makes it possible to avoid the need for human donors and human products thereby decreasing the risk of disease transmission, protein impurities and batch-to-batch inconsistencies. Many monoclonal and recombinant anti-D $\mathrm{IgG}$ have been developed and tested. Most of these comprise of IgG1 antibodies which is the dominant $\operatorname{IgG}$ sub-class in the polyclonal preparation (about 9:1 IgG1:IgG3). Some of these have been tested clinically [12]. Certain monoclonal anti-D preparations are currently being studies in early phases of clinical trials $[11,13]$.

Rhoclone ${ }^{\circledR}$ is a preparation of human monoclonal antirhesus antibodies (IgG1 subclass) that is available in India since 2007 and is to date the only monoclonal anti-D marketed in the world. It is derived from a stable heterohybridoma cell line and purified using protein affinity chromatography [14]. This is the only study to have evaluated the application of monoclonal anti-D in a large number of women requiring anti-D in the clinical setting. The study did not evaluate antenatal administration of anti-D as it would have impacted study conduct and analysis 1) many of the enrolled subjects would have ended up with Rh-negative baby, thus not being ideal candidates for efficacy assessment and 2) ensuring that subjects enrolled in the antenatal period return to the same study site for child-birth and receive the same anti-D brand would have been a challenge.

In this study, both monoclonal and polyclonal anti-D IgG preparations demonstrated effective protection in Rh-negative women, against isoimmunization with $\mathrm{Rh}$ antigen from their Rh-positive babies. One patient in the polyclonal group was ICT positive. Despite the use of postnatal prophylaxis, antibody formation occurs during pregnancy in about $1-2 \%$.
Even with addition of antenatal prophylaxis, the rate of antibody formation is $0.2-0.3 \%$ but not $0 \%$ [15]. Alternatively, the dosage could have been inadequate. The standard dose of $300 \mathrm{mcg}$ for prophylaxis will protect 997/1000 women from isoimmunisation; in cases with fetomaternal haemorrhage, this dose could prove inadequate [16].

No specific adverse reactions related to either preparation was reported. In addition, the monoclonal anti-D preparation did not result in an immunogenic reaction in any subject. Coupled with the other advantages associated with monoclonal antibodies over polyclonal antibodies-better batchto-batch consistency, lesser protein impurities, lesser risk of disease transmission and practically unlimited supply, monoclonal anti-D provides an attractive and viable alternative to the conventional polyclonal preparation.

\section{Conclusion}

In conclusion, the study demonstrated that the two anti-D preparations are clinically similar, and the newer monoclonal anti-D preparation is a suitable alternative to the conventional polyclonal anti-D in the prevention of maternal isoimmunization.

\section{Compliance with Ethical Standards}

Ethics The trial protocol was approved by the Indian drug regulatory authority CDSCO (Central Drugs Standard Control Organization). The trial protocol was approved by the institutional ethics committees of all nine participating centres individually. The trial was registered with the CTRI (Clinical Trials Registry - India) prior to commencement. The trial was performed in accordance with the principles of the Declaration of Helsinki, the International Conference on Harmonization Guidelines for Good Clinical Practice, and local regulatory requirements.

Conflict of interest The authors declare that they have no conflict of interest, nor do they have any commercial association or financial interest in the publication of this manuscript. The authors take complete responsibility for the integrity of the data and the accuracy of the data analysis.

Informed Consent Informed consent was obtained from all individual participants included in the study by the respective investigators at each study site.

Funding The clinical study was unconditionally supported financially and materially by Bharat Serums and Vaccines Limited.

\section{References}

1. Kent J, Farrell AM, Soothill P. Routine administration of AntiD: the ethical case for offering pregnant women fetal RHD 
genotyping and a review of policy and practice. BMC Pregnancy Childbirth. 2014;14(1):87.

2. Bolton-Maggs PH, Davies T, Poles D, et al. Errors in anti-D immunoglobulin administration: retrospective analysis of 15 years of reports to the UK confidential haemovigilance scheme. BJOG Int J Obstet Gynaecol. 2013;120(7):873-8.

3. Olovnikova N. Anti-RhD-mediated immunosuppression: can monoclonal antibodies imitate the action of polyclonal antibodies?. In: Immunosuppression-role in health and diseases 2012. InTech.

4. McBain RD, Crowther CA, Middleton P. Anti-D administration in pregnancy for preventing Rhesus alloimmunisation. Cochrane Database Syst Rev. 2015;9(2):CD000020.

5. Dajak S, Roje D, Hašpl ŽH, et al. The importance of antenatal prevention of $\mathrm{RhD}$ immunisation in the first pregnancy. Blood Transfus. 2014;12(3):410.

6. De Haas M, Thurik FF, Van Der Ploeg CP, et al. Sensitivity of fetal RHD screening for safe guidance of targeted anti-D immunoglobulin prophylaxis: prospective cohort study of a nationwide programme in the Netherlands. BMJ. 2016;7(355):i5789.

7. Velkova E. Correlation between the amount of anti-D antibodies and IgG subclasses with severity of haemolytic disease of foetus and newborn. Open Access Maced J Med Sci. 2015;3(2):293.

8. Fyfe TM, Ritchey MJ, Taruc C, et al. Appropriate provision of anti-D prophylaxis to $\mathrm{RhD}$ negative pregnant women: a scoping review. BMC Pregnancy Childbirth. 2014;14(1):411.

9. Costumbrado J, Ghassemzadeh S. Rh Incompatibility. [Updated 2017 Oct 16]. In: StatPearls [Internet]. Treasure Island (FL): StatPearls Publishing; 2018 Jan. https://www.ncbi.nlm.nih.gov/books /NBK459353/.

10. European Medicines Agency. Committee for Medicinal Products for Human Use-Guideline on the clinical investigation of human anti-D immunoglobulin for intravenous and/or intramuscular use. CPMP/BPWG/575/99 Rev. 1. https://www.ema.europa.eu/docum ents/scientific-guideline/guideline-clinical-investigation-human -anti-d-immunoglobulin-intravenous/intramuscular-use_en.pdf. Accessed 2 Sept 2018.

11. Prevention of Rh D alloimmunization. Practice Bulletin No. 181 American College of Obstetricians and Gynecologists. Obs Gynecol. 2017;130:e57-70.

12. Kumpel BM. Efficacy of RhD monoclonal antibodies in clinical trials as replacement therapy for prophylactic
anti-D immunoglobulin: more questions than answers. Vox Sang. 2007;93(2):99-111.

13. Reichert JM. Antibodies to watch in 2017. In: MAbs 2017 Feb 17, vol 9, no. 2. Taylor \& Francis. p. 167-81.

14. Rhoclone Pack Insert. [cited 2018 Mar 30]. www.bharatseru $\mathrm{ms} . \mathrm{com} /$ product/endoprost/RHOCLONE(Liquid)CommonPack InsertforDomestic.pdf.

15. Qureshi H, Massey E, Kirwan D, et al. BCSH guideline for the use of anti-D immunoglobulin for the prevention of haemolytic disease of the fetus and newborn. Transfus Med. 2014;24(1):8-20.

16. Ramsey G. Inaccurate doses of Rh immune globulin after Rhincompatible fetomaternal hemorrhage: survey of laboratory practice. Arch Pathol Lab Med. 2009;133(3):465-9.

Publisher's Note Springer Nature remains neutral with regard to jurisdictional claims in published maps and institutional affiliations.

\section{About the Author}

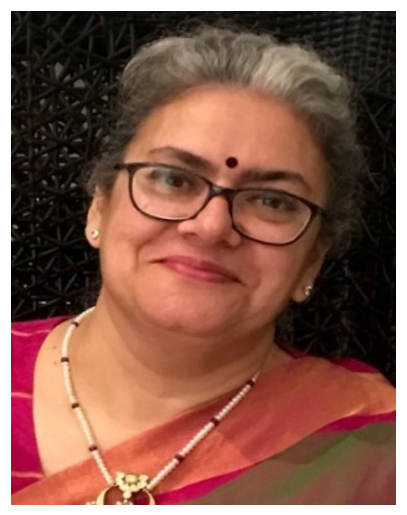

Dr. Anahita Chauhan Dr. Anahita Chauhan is former Professor and Unit Head in the Department of Obstetrics and Gynaecology at Seth G. S. Medical College and K.E.M. Hospital in Mumbai, India. She is Honorary Consultant at Saifee and St. Elizabeth Hospitals, Mumbai. She serves as Second Joint Assistant Editor of the Journal of Obstetrics and Gynecology of India and as Office Bearer (Treasurer) of the Mumbai Obstetric and Gynaecological Society.

\section{Affiliations}

\section{Anahita R. Chauhan ${ }^{1} \cdot$ Yogeshwar S. Nandanwar ${ }^{2} \cdot$ Aruna Ramaiah $^{3} \cdot$ Kanan A. Yelikar $^{4} \cdot$ M. D. Rashmi ${ }^{5}$. Rekha Sachan $^{6} \cdot$ Rahul V. Mayekar $^{2} \cdot$ Yamini N. Trivedi $^{7} \cdot$ Gopalkrishna V. Paradkar $^{8} \cdot$ Kiran P. Patole $^{9}$}

1 Department of Obstetrics and Gynaecology, Seth GS Medical College and King Edward Memorial Hospital, Mumbai, Maharashtra 400012, India

2 Department of Obstetrics and Gynaecology, Lokmanya Tilak Municipal Medical College and General Hospital, Mumbai, Maharashtra 400022, India

3 Department of Obstetrics and Gynaecology, Modern Government Maternity Hospital, Hyderabad, Telangana 500064, India

4 Department of Obstetrics and Gynaecology, Government Medical College and Hospital, Aurangabad, Maharashtra 431004, India
5 Department of Obstetrics and Gynaecology, Apollo BGS Hospitals, Mysuru, Karnataka 570023, India

6 Department of Obstetrics and Gynaecology, King George's Medical University, Lucknow, Uttar Pradesh, 226003, India

7 Department of Obstetrics and Gynaecology, Sheth L.G. General Hospital, Ahmedabad, Gujarat 380008, India

8 Department of Obstetrics and Gynaecology, Rajiv Gandhi Medical College and Chhatrapati Shivaji Maharaj Hospital, Thane, Maharashtra 400605, India

9 Department of Obstetrics and Gynaecology, Dr. Vasantrao Pawar Medical College, Hospital and Research Centre, Nashik, Maharashtra 422207, India 